\title{
Impact of pad material on stability of tool influnce function in CCOS
}

\author{
Bo Zhong ${ }^{a}$, Xianhua Chen ${ }^{b}$ and Jie $\mathrm{Li}^{\mathrm{c}}$ \\ Research Center of Laser Fusion, China Academy of Engineering Physics \\ No.3 Keyuan 1st Road, Hi-Tech Zone, Chengdu China \\ a zhongbo_foerc@163.com, ${ }^{b}$ chenmail2@163.com, jennylj1103@126.com
}

\begin{abstract}
Keywords: Computer controlled optical surfacing, Tool influnce function, Pad material Abstract. The stability of tool influnce function (TIF) is the key to achieve deterministic removal in the computer controlled optical surfacing (CCOS). In this paper, the comparative experiment was conducted to investigate the impact of two common pad materials (i.e., cloth, pitch) on the TIF. In addition, by assistant of the dynamometer, the polishing force of the TIF was detected to reveal the reason of the instability of TIFs. The results show that: 1) the stability of cloth tool is better than that of pitch tool, and the stability of TIFs peak removal efficiency with cloth and pitch are $93.5 \%$ and $76 \%$, respectively. 2) the stability of TIFs are directly influenced by the frictional force, which indirectly reflects the stability of the contact state of the workpiece and polishing tool. The research of this paper also provides an important foundation for the further study of improving material removal stability based on the optimization of pad material.
\end{abstract}

\section{Introduction}

In order to meet the requirements of high precision and mass production of optical components, the fabricating process of "ultra precision grinding and deterministic polishing" has been put forward. Indeed, in the deterministic polishing process, the surface error of the optical component is corrected to meet the final quality of the surface. The fabricating efficiency and precision of optical components is determinated by the deterministic polishing. Because of the importance of the deterministic polishing process, the related research has attracted much attention and developed rapidly. At present, the computer controlled optical surfacing (CCOS) based on different sub-aperture polishing tools is supposed to be the most frequently used technology in the deterministic polishing process. However, there is a wildly recognized challenge in this technology, i.e., the instability of TIF, which causes by some factors, such as the distortion of tool surface, the variety of tool rigidity. In CCOS, the unstable TIF will lead to the uncertainty in the material removal, and reduce the fabricating precision and efficiency of optical components [1-4].

In the existing theories of material removal in CCOS, it is a premise that the TIF maintains stable. However, many researches pointed out that the variety of the tool polishing performance will affect the stability and certainty of material removal, and then influence on the producing efficiency and accuracy of the optics. Ando [5] indicated that by using the special production equipment of pitch tool, the surface shape of pitch tool was accurately controlled, and combining the optimized process parameters, the fluctuate of TIFs in 10 experiments is less than $10 \%$ for peak removal efficiency, and is less than 6\% for the volume removal efficiency. Dai [6] analyzed the influence of the pad materials on the removal efficiency and material removal stability, and indicated that the tool wear had an important influence on the removal stability.

The sub-aperture polishing technology based on pitch tool is easy to obtain smooth surface, which is the most widely used polishing technology in engineering production, but the actual processing reflects the poor certainty of pitch tool processing. This paper studies the impact of pad materials on the stability of TIF, and discuss the reason of the instability of TIFs from the frictional force. 


\section{Experimental design and setup}

With the aim to investigate the impact of pad material on the stability of TIF, comparative experiment was carried out in this section. Indeed, two kinds of pads (i.e., pitch and cloth) are applied directly to polish the specimens under the same conditions, and the removal stability are compared and discussed. The experiment was designed as follows. The research methods adopted in this paper is that based on the TIFs of different pad materials and the polishing force, the relationship between frictional force and removal characteristic was analyzed, and then the impact of pad material on the stability of TIF was revealed.

Fig. 1 illustrates the principle and the setup of the measurement of polishing forces during polishig process. As can be seen, the removal process is conducted on a $\Phi 320 \mathrm{~mm} \mathrm{sio}_{2}$ glass, the peak-to-valley value (PV) of which is pre-polished to $\sim 0.5 \mu \mathrm{m}$. Other experimental conditions are listed in Table 1. Besides, for each, six spots are polished. Meanwhile, during the polishing process, the polishing forces on the workpiece containing the normal force $\left(F_{n}\right)$ and the tangential force (i.e., the tangential force in $\mathrm{x}$ direction $F_{t x}$, the tangential force in y direction $F_{t y}$ ) are collected by a 3-components dynamometer (model-9257B, Kistler made). After the polishing process, the spots are measured by a interferometer(model- ASI(Q),QED made), therefore the tool removal characteristic includes the removal efficiency and the shape of spots are achieved.

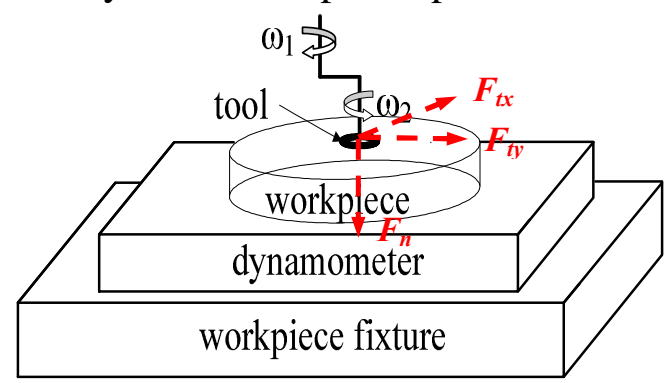

(a)

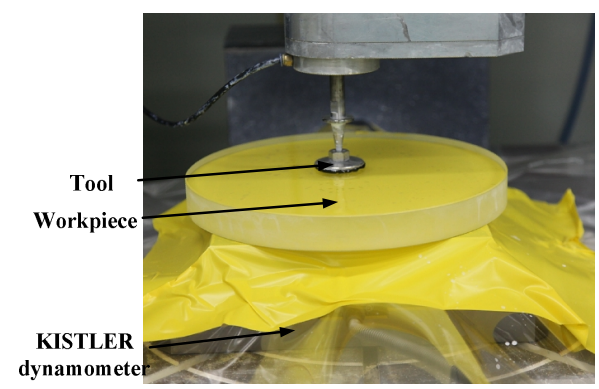

(b)

Fig. 1 The principle and the setup of the measurement of polishing forces during polishig

Table 1 Experimental conditions

\begin{tabular}{c|c|c|c|c}
\hline $\begin{array}{c}\text { Tool radius } \\
(\mathrm{mm})\end{array}$ & $\begin{array}{c}\text { Revolution radius } \\
(\mathrm{mm})\end{array}$ & $\begin{array}{c}\text { Rotational speed } \omega_{1} \\
(\mathrm{rpm})\end{array}$ & $\begin{array}{c}\text { revolution speed } \omega_{2} \\
(\mathrm{rpm})\end{array}$ & $\begin{array}{c}\text { time } \\
\left({ }^{\circ}\right)\end{array}$ \\
\hline 25 & 5 & 20 & 200 & 420 \\
\hline
\end{tabular}

\section{Results and Discussion}

Fig. 2 (a) (b) shows the contour map and depth map of two kinds of polishing tools. According to Fig. 2, it can be seen that the shapes of TIFs with two tools are different under the same experimental conditions. The details of the polishing spots (TIF) in Fig. 2 are shown in Table 2, which includes the full width at half maximum (FWHM), the peak removal depth, peak removal efficiency and volume removal efficiency, and Fig. 3 shows the corresponding comparison results.
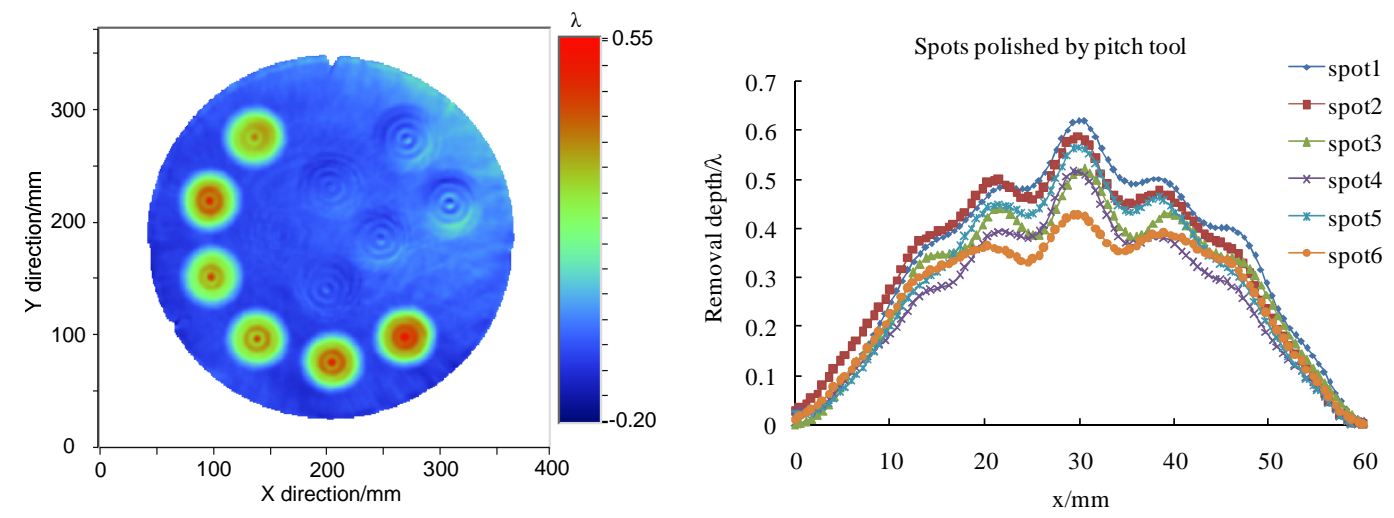

(a) 

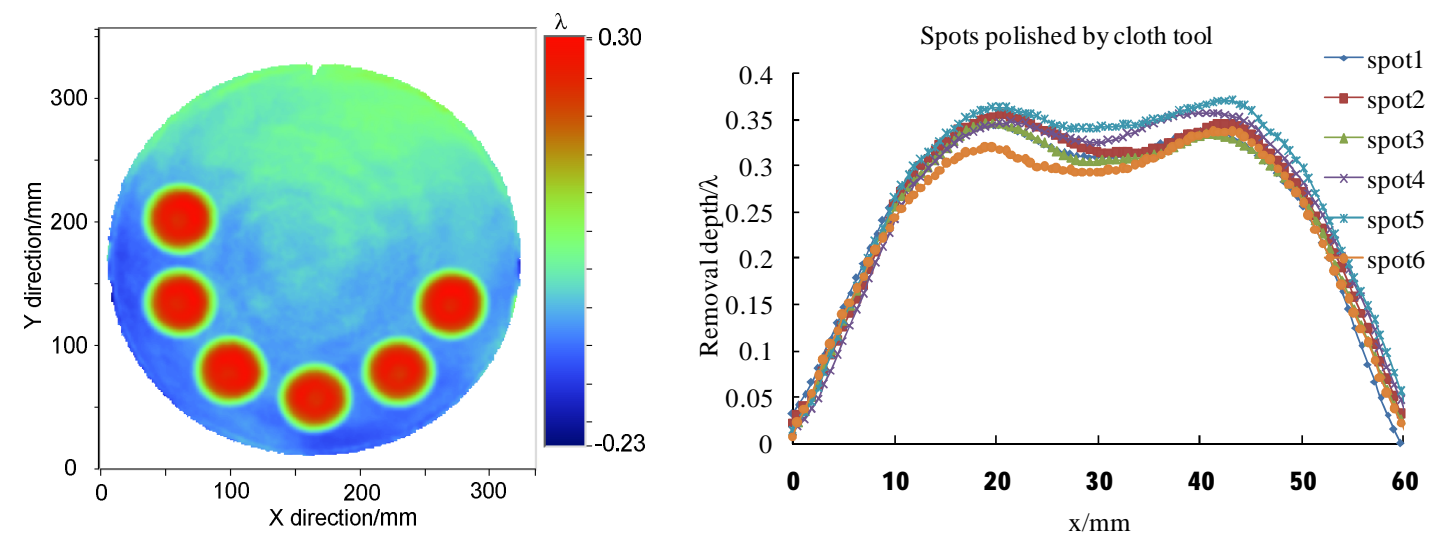

(b)

Fig. 2 Spots polished by two tools

Table 2 Removal characteristic with two tools

\begin{tabular}{|c|c|c|c|c|c|}
\hline $\begin{array}{l}\text { Tool } \\
\text { type }\end{array}$ & $\begin{array}{c}\text { Spot } \\
\text { size } \\
(\mathrm{mm})\end{array}$ & $\begin{array}{l}\text { Full width at half } \\
\text { maximum (mm) }\end{array}$ & $\begin{array}{l}\text { Peak removal } \\
\text { depth }(\lambda)\end{array}$ & $\begin{array}{c}\text { Peak removal } \\
\text { efficiency }(\mathrm{nm} / \mathrm{s})\end{array}$ & $\begin{array}{c}\text { Volume Removal } \\
\text { efficiency } \\
\left(\mathrm{mm}^{3} / \mathrm{min}\right)\end{array}$ \\
\hline \multirow{6}{*}{$\begin{array}{c}\text { Pitch } \\
\text { tool }\end{array}$} & \multirow{12}{*}{60} & 38.40 & 0.6258 & 0.9429 & 0.0710 \\
\hline & & 37.80 & 0.6006 & 0.9049 & 0.0655 \\
\hline & & 39.01 & 0.5376 & 0.8100 & 0.0629 \\
\hline & & 37.18 & 0.5418 & 0.8163 & 0.0572 \\
\hline & & 38.40 & 0.5880 & 0.8859 & 0.0654 \\
\hline & & 41.45 & 0.4200 & 0.6328 & 0.0562 \\
\hline \multirow{6}{*}{$\begin{array}{c}\text { Cloth } \\
\text { tool }\end{array}$} & & 43.80 & 0.3688 & 0.5556 & 0.0596 \\
\hline & & 43.18 & 0.3625 & 0.5461 & 0.0574 \\
\hline & & 43.18 & 0.3881 & 0.5847 & 0.0632 \\
\hline & & 45.00 & 0.3746 & 0.5645 & 0.0595 \\
\hline & & 43.80 & 0.4049 & 0.6100 & 0.0652 \\
\hline & & 44.45 & 0.3826 & 0.5765 & 0.0625 \\
\hline
\end{tabular}

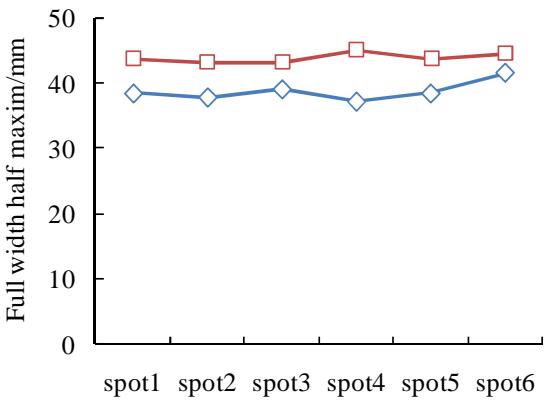

(a) Full width at half maximum

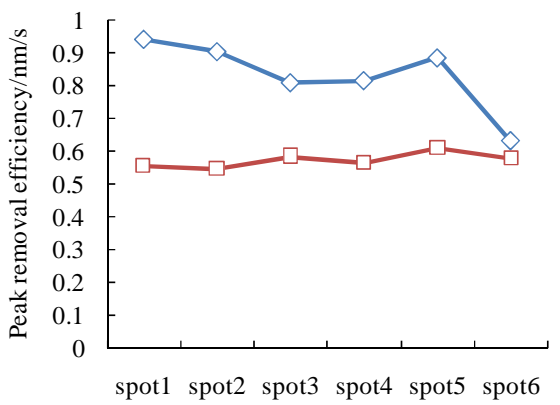

(c) Peak removal efficiency

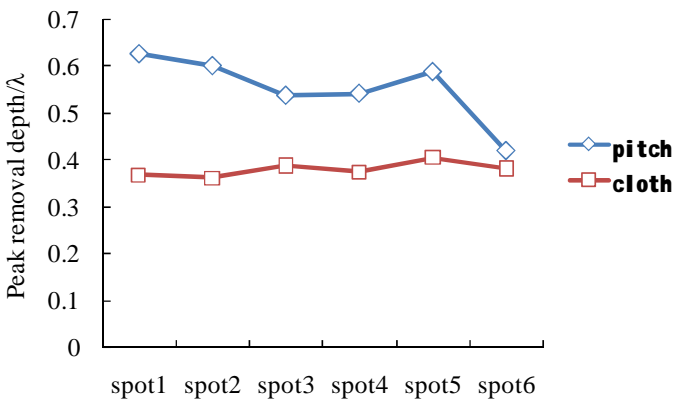

(b) Peak removal depth

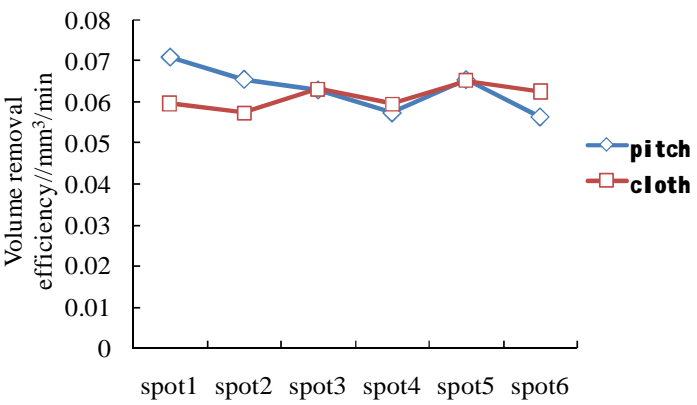

(d) Removal volume efficiency

Fig. 3 Comparison results of pitch tool and cloth tool

Based on the above experimental results, this section will discuss the influence of polishing tool materials on removal characteristics from two aspects, i.e., spot shape, removal efficiency. 


\section{(1) spot shape}

As shown in Fig. 2, the shape of TIF with pitch tool is approximately cone-shaped, and the shape of TIF with cloth tool is approximately gibbose. As shown in Table 1, the FWHM of TIFs with cloth tool and pitch tool (average value) are $38.7 \mathrm{~mm}$ and $43.9 \mathrm{~mm}$, respectively, and the fluctuate of FWHM of TIFs are $7 \%$ (the stability 93\%) and $2.5 \%$ (the stability $97.5 \%$ ), respectively. The experimental results show that the TIF of cloth tool is more stable than that of pitch tool.

(2) removal efficiency

From Fig.3 (b) (c), it can be seen that the fluctuate of the removal depth and peak removal efficiency with pitch tool is intense, and the fluctuation amount is $24 \%$ (the stability $76 \%$ ); while with cloth tool, the fluctuation amount is $6.5 \%$ (the stability 93.5\%). In terms of volumetric removal efficiency, as shown in Fig. 3 (d), the average volume removal efficiency of pitch tool and cloth tool are $0.063 \mathrm{~mm}^{3} / \mathrm{min}$ and $0.061 \mathrm{~mm}^{3} / \mathrm{min}$, respectively, and their efficiency fluctuations are $12.5 \%$ (the stability $87.5 \%$ ) and $6.5 \%$ (the stability 93.5\%), respectively. The above results show that the removal efficiency of pitch tool and cloth tool is closer, but there is a big difference in stability, and the stability of cloth tool is better than the stability of pitch tool.

It is well known that the Preston law, i.e., $\mathrm{R}=\mathrm{k}^{*} \mathrm{p}^{*} \mathrm{v}$, is the most frequently used theory to explain the material removal mechanism of polishing process. In which, $\mathrm{R}$ represents the removal amount of the material, $\mathrm{k}$ is the Preston coefficient, $\mathrm{p}$ and $\mathrm{v}$ are the pressure and velocity of the polishing spot. In the above experiment, expect the pad material, the experimental conditions were consistent. Therefore, the velocity distribution of two kinds of polishing tool is consistent in contact area with the workpiece, but that of contact state between polishing tool and workpiece is not stable, resulting in $\mathrm{P}$ and $\mathrm{K}$ in the formula is not stable, thereby affecting the stability of TIF (shape and efficiency). The contact state between the polishing tool and the workpiece is related to the factors, i.e., the pad material, the asperity distribution of the pad and the hardness of the polishing material. The above factors were hardly detected and characterized separately in the experiment. As the variety of the contact state, the polishing force (frictional force) will be changed directly. Therefore, in the experiment, the frictional force in the polishing process was detected. According to the fluctuation state of the friction force, the variety of the factors, i.e., the pad material, the asperity distribution of the pad and the hardness of the polishing material. were comprehensively reflected. The frictional forces in the polishing process were measure and indicated in Fig. 4, Fig. 5.

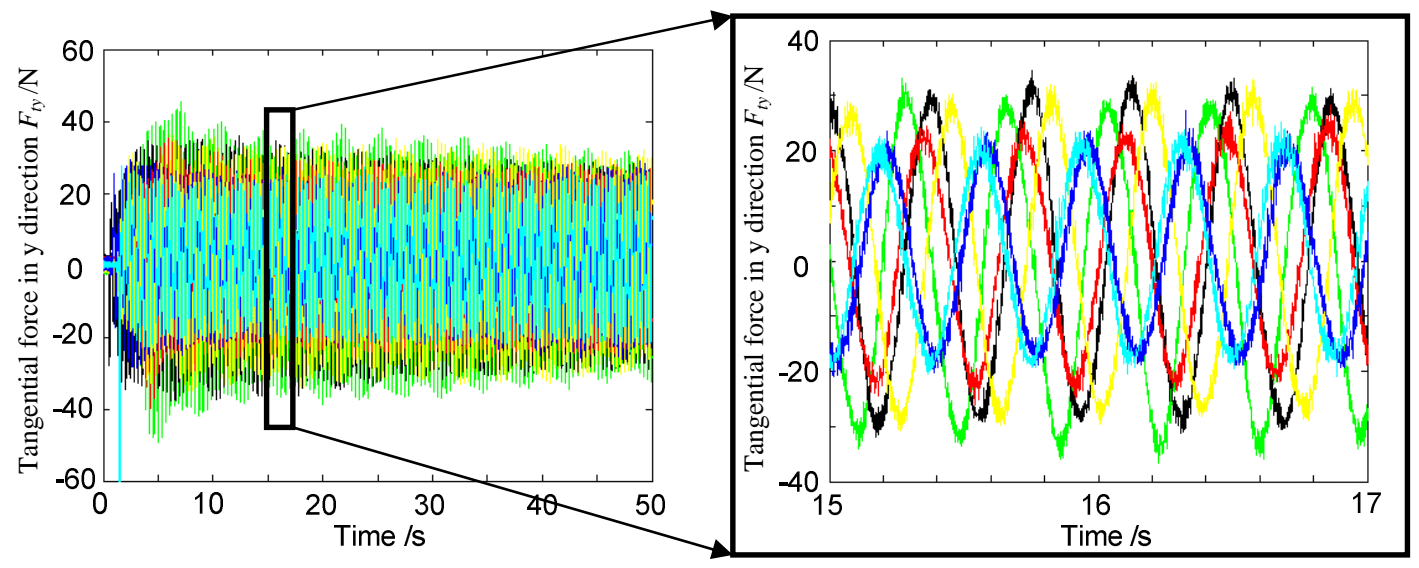

Fig.4 Tangential force in y direction with pitch tool 


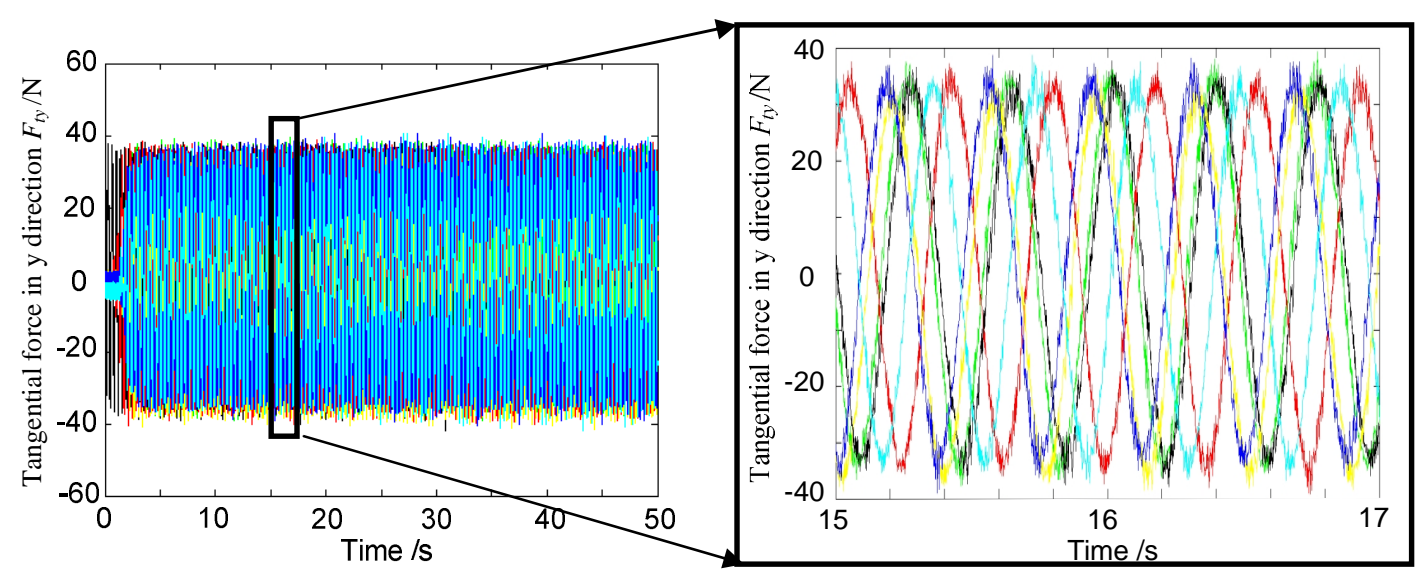

Fig.5 Tangential force in y direction with cloth tool

Fig.4 shows the tangential force (frictional force) in y direction with pitch tool. The left picture shows the results of $F_{t y}$ for $50 \mathrm{~s}$, and the right picture shows the the results $F_{t y}$ from $15 \mathrm{~s}$ to $17 \mathrm{~s}$. In addition, the curves of different colors represent the tangential forces of collecting different spots. It is shown that the tangential force is the periodic variation, and the period is about $0.375 \mathrm{~s}$, which is related to the revolution speed of the polishing tool. The peak and valley (PV) of the polishing forces are obvious differences. The minimum value, maximum value and average value of PV are about $40 \mathrm{~N}$, $60 \mathrm{~N}$ and $52.7 \mathrm{~N}$, respectively. Therefore, the fluctuation of the frictional force with pitch tool is about $24 \%$ (the stability 76\%). Fig.5 shows the tangential force (frictional force) in y direction with cloth tool. According to Fig. 5, the difference of the polishing forces is very small. And, the minimum value, maximum value and average value of PV is about $70 \mathrm{~N}, 78 \mathrm{~N}$ and $74 \mathrm{~N}$. Therefore, the fluctuation of the frictional force with cloth tool is about $5.4 \%$ (the stability 94.6\%). The above results show that the frictional force of cloth tool is larger than that of pitch tool. However, Fig. 3 (b) showed that the peak removal efficiency of cloth tool is lower than that of pitch tool, which means that the preston coefficient $\mathrm{K}$ of cloth tool is lower than that of pitch tool. In addition, the comparison of the frictional force with two tools, the stability of the cloth tool is better than that of the pitch tool in the polishing process. Furthermore, the comparison of the fluctuation of the frictional force and the removal efficieny shows that the stability of TIFs with different pad materials are directly influenced by the polishing force, which indirectly reflects the stability of the contact state of the workpiece and polishing materials. According to the above results, relative to the pitch tool, the cloth tool has excellent stability and adaptability, which easily fits with workpiece and is hard worn.

\section{Conclusions}

To investigate the impact of the pad material on the stability of TIF in CCOS, the TIFs with cloth tool and pitch tool was achieved and analyzed. In addition, the polishing force was detected to reveal the reason of the instability of TIFs. The following conclusions are obtained:

1) The stability of TIF is evaluated from three aspects, i.e., the FWHM, the peak removal efficiency and the volume removal efficiency. In the FWHM of TIF, the stability of TIF with cloth tool and pitch tool are $97.5 \%$ and $93 \%$, respectively. In the peak removal efficiency of TIF, the stability of TIF with cloth tool and pitch tool are $93.5 \%$ and $76 \%$, respectively. In the volume removal efficiency of TIF, the stability of TIF with cloth tool and pitch tool are $93.5 \%$ and $87.5 \%$, respectively. In a word, the stability of TIF with cloth tool is more stable than that of TIF with pitch tool.

2) In the case of maintaining the normal force and other experimental conditions, the stability of frictional force with cloth tool and pitch tool are $94.6 \%$ and $76 \%$, respectively. Therefore, the stability of the frictional force with cloth tool is better than that of cloth tool. The variety of polishing force is same to that of TIF, which reveals that stability of TIFs are directly influenced by the frictional force, which indirectly reflects the stability of the contact state of the workpiece and polishing tool. 


\section{Acknowledgements}

This work was financially supported by the Science Challenge Project (JCKY2016212A506-0502), the Youth Talent Fund of Laser Fusion Research Center, CAEP. (RCFCZ1-2017-6) and the Laboratory of Precision Manufacturing Technology, CAEP. (ZZ14006).

\section{References}

[1] P. K. Mehta and R. E. Hutnagel. Pressure distribution under flexible polishing tools 1Conventional aspheric optics. Proc. SPIE, Vol. 1303(1990), pp. 178-188.

[2] P. K. Mehta. Pressure distribution under flexible polishing tools -2 Cylindrical (conical) optics. Proc. SPIE, Vol. 1303 (1990), pp. 189-205.

[3] J. H. Burge, B. Anderson, S. Benjamin, et al. Development of optimal grinding and polishing tools for aspheric surfaces. Proc. SPIE, Vol. 4451 (2001), pp. 153-164.

[4] U. Birnbaum, H. Bernitzki, O. Falkenstörfer, et al. Manufacturing of high-precision Aspheres. Proc. SPIE, Vol. 6149 (2006), pp. 61490H-1-61490H-7.

[5] M. Ando, M. Negishi, M.Takimoto. Super-smooth polishing on aspherical surfaces(II) Achievement of a super-smooth polishing. Proc. SPIE, Vol. 2576 (1995), pp. 348-356.

[6] Y. F. DAI, W. J. SHANG, X. S. ZHOU. Effection of the Material of a Small Tool to the Removal Function in Computer Control Optical Polishing. Journal of national university of defence technology, Vol. 28, No. 2 (2006), pp. 97-101. 